\title{
Aminolysis of iridium thiophene compounds
}

\section{Angeles Paz-Sandoval,* Marisol Cervantes-Vásquez, and Brenda A. Paz-Michel}

Departamento de Química, Cinvestav, Av. IPN \# 2508 esq. Ticomán, San Pedro Zacatenco, México 07360, D.F. México

E-mail: mpaz@cinvestav.mx

\section{Dedicated to Professor Rosalinda Contreras, friend and colleague, on the ocassion of her $6^{\text {th }}$ birthday in recognition of her oustanding contribution to the main group chemistry}

\begin{abstract}
The dicationic iridium 2,5-dimethylthiophene $\mathrm{Cp} * \operatorname{Ir}[\mathrm{SC}(\mathrm{Me}) \mathrm{CHCHC}(\mathrm{Me})]\left[\mathrm{BF}_{4}\right]_{2}$ (6) reacts with secondary cyclic and acyclic amines to give salts with formula $\left[\mathrm{Cp} * \operatorname{Ir}\left[\mathrm{SC}(\mathrm{Me}) \mathrm{CHCH}(\mathrm{Me}) \mathrm{C}=\mathrm{NR}_{2}\right][\mathrm{BF} 4]\left[\mathrm{R}_{2}\right.\right.$ = piperidyl, (12); pyrrolidyl, (13); morpholyl, (14); piperazyl, (15); $\mathrm{R}=$ diethyl (16) and methyl, benzyl (17)]. The coordinated thiophene compound undergoes $\mathrm{C}$-S cleavage to give the corresponding iminium-thiolate derivatives. The hydrolysis of the iminium salts affords the acylthiolate $\mathrm{Cp}^{*} \operatorname{Ir}[\mathrm{SC}(\mathrm{Me}) \mathrm{CHCH}(\mathrm{Me}) \mathrm{C}=\mathrm{O}](7)$.

The characterization of 12-17 is established in solution through ${ }^{1} \mathrm{H},{ }^{11} \mathrm{~B},{ }^{13} \mathrm{C}$ and ${ }^{19} \mathrm{~F} \mathrm{NMR}$, and compounds 12-14 are also studied in the solid state by X-ray diffraction. The crystalline structures, as well as the studies in solution for compounds 12-17, show only one thermodynamic product, which was obtained from previous isomerization of the kinetic species; meanwhile compound 16 was obtained in mixture with 7 and the corresponding quaternary salt.
\end{abstract}

Keywords: Thiophene, iridium, nucleophilic addition, aminothiols

\section{Introduction}

Dicationic ruthenium compounds $[(\text { arene }) \mathrm{Ru}(\text { thiophene })]^{2+}$ containing thiophene, 2methylthiophene, 2,5-dimethylthiophene and 1,2,3,4-tetramethylthiophenes react with ammonia to give salts of the formula $\left[(\right.$ arene $\left.) \mathrm{Ru}\left(\mathrm{SC}_{4} \mathrm{R}_{4} \mathrm{NH}_{2}\right)\right] \mathrm{X}$ (arene $=\mathrm{C}_{6} \mathrm{Me}_{6}$ or cymene; $\mathrm{R}=\mathrm{H}, \mathrm{Me} ; \mathrm{X}$ $=\mathrm{BF}_{4}, \mathrm{PF}_{6}$ or OTf). The coordinated thiophene, as well as the substituted 2- and 2,5-methyl derivatives undergo $\mathrm{C}-\mathrm{S}$ cleavage in order to give the corresponding iminium thiolate derivatives. $^{1}$

Additional stereochemical insights into the ammonolysis reaction are obtained by studies on the 2,5-dimethylthiophene ruthenium compound $\left[(\text { cymene }) \mathrm{Ru}\left(2,5-\mathrm{Me}_{2} \mathrm{C}_{4} \mathrm{H}_{2} \mathrm{~S}\right)\right]^{2+}$ (1) where there 
is evidence of a kinetic $\mathbf{2}$ and thermodynamic $\mathbf{3}$ isomers (Scheme1a). The isomerization occurs under very mild conditions and there is no evidence of racemization. The kinetic isomer can be rearranged to the thermodynamic one upon standing, even at solid state, at $30^{\circ} \mathrm{C} .{ }^{1}$

Solutions of $\left[(\right.$ cymene $\left.) \mathrm{Ru}\left\{\eta^{4}-\mathrm{SC}(\mathrm{Me}) \mathrm{CHCHC}(\mathrm{Me}) \mathrm{NH}_{2}\right\}\right] \mathrm{OTf}(\mathbf{2})$ react with aqueous $\mathrm{KOH}$ in order to give the kinetic isomer of the corresponding acylthiolate [(cymene) $\mathrm{Ru}\left\{\eta^{4}\right.$ $\mathrm{SC}(\mathrm{Me}) \mathrm{CHCHCO}(\mathrm{Me})\}]$ (4), Scheme 1a. The ammonolysis of 1,2,3,4-tetramethylthiopene complexes proceeds rather differently from the cases of the less substituted thiophenes. ${ }^{1}$

Base hydrolysis of dicationic (cymene) $\mathrm{Ru}(\mathrm{II})$ and $\mathrm{Cp} * \mathrm{Ir}(\mathrm{III})$ with the coordinated 2,5dimethyl-thiophene, which has also been studied, results in the generation of the corresponding acylthiolate derivatives [(cymene) $\left.\mathrm{Ru}\left\{\eta^{4}-\mathrm{SC}(\mathrm{Me}) \mathrm{CHCHCOMe}\right\}\right] \quad(4)^{2}$ and $\left[\mathrm{Cp} * \operatorname{Ir}\left\{\eta^{4}-\right.\right.$ $\mathrm{SC}(\mathrm{Me}) \mathrm{CHCHCOMe}\}](7),{ }^{3,4}$ respectively. The acylthiolate derivatives react to afford more complex polinuclear compounds: dinuclear (5) in the case of ruthenium, ${ }^{2}$ (Scheme 1a) and dinuclear $(\mathbf{9}, \mathbf{1 0})$ and tetranuclear $(\mathbf{8})$ in the case of iridium ${ }^{3}$ compounds, (Scheme 1b).

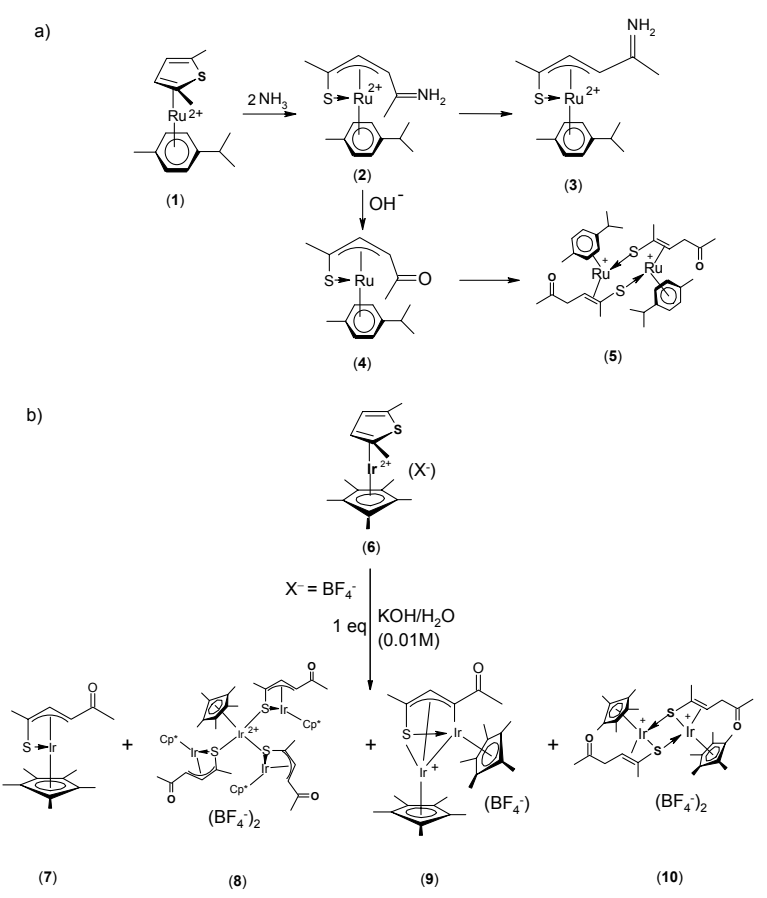

\section{Scheme 1}

Thus in order to expand our knowledge concerning the behavior of compound $\mathbf{6}$, we decided to perform a thorough investigation of the chemical properties of this compound in the presence of secondary amines. 


\section{Results and Discussion}

The addition of secondary amines to $[\mathrm{Cp} * \operatorname{Ir}(2,5$-dimethyl-thiophene $)]\left[\mathrm{BF}_{4}\right](\mathbf{6})$ resulted in a rapid color change from white to yellow, orange or red, for compounds $\left[\mathrm{Cp} * \mathrm{Ir}\left\{\mathrm{SC}(\mathrm{Me}) \mathrm{CHCH}(\mathrm{Me}) \mathrm{C}=\mathrm{NR}_{2}\right\}\right] \mathrm{BF}_{4}\left[\mathrm{R}_{2}=\right.$ piperidyl (12); pyrrolidyl (13); morpholyl (14); piperazyl (15)], which were obtained after stirring $18 \mathrm{~h}$ at room temperature, Scheme 2.

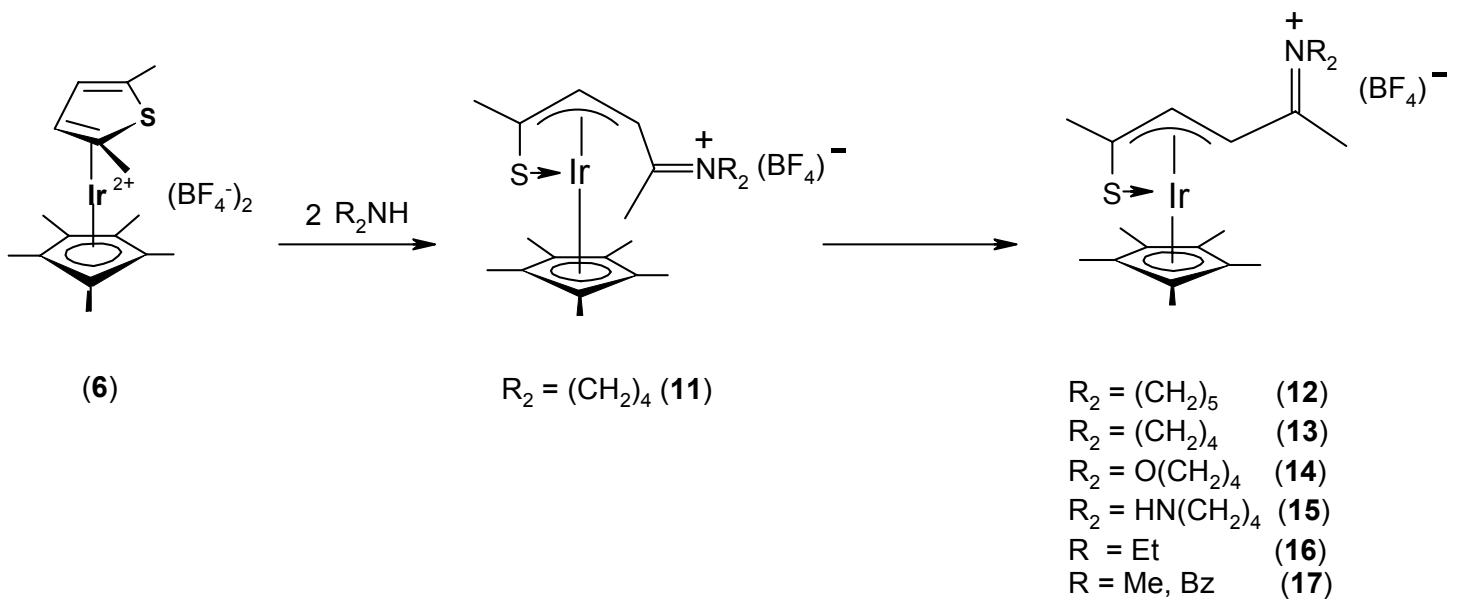

\section{Scheme 2}

The reaction was so well behaved for cyclic amines, it gave the iminium salts in good yields; meanwhile diethylamine, which is an acyclic derivative, showed that there was a competition between the formation of compound $\left[\mathrm{Cp} * \operatorname{Ir}\left\{\mathrm{SC}(\mathrm{Me}) \mathrm{CHCH}(\mathrm{Me}) \mathrm{C}=\mathrm{N}(\mathrm{Et})_{2}\right\}\right] \mathrm{BF}_{4}(\mathbf{1 6})$ and its hydrolysis to afford the acylthiolate compound $[\mathrm{Cp} * \operatorname{Ir}\{\mathrm{SC}(\mathrm{Me}) \mathrm{CHCH}(\mathrm{Me}) \mathrm{C}=\mathrm{O}\}](7)$, as well as its corresponding quaternary salt $\left[\mathrm{NH}_{2}(\mathrm{Et})_{2}\right] \mathrm{BF}_{4}$. Diisopropylamine and dicyclohexylamine showed the formation of compound 7 along with the corresponding quaternary salt $\left.\left[\mathrm{NH}_{2}(\mathrm{R})_{2}\right] \mathrm{BF}_{4}\left[\mathrm{R}=\mathrm{CHMe}_{2} ; \mathrm{C}_{6} \mathrm{H}_{11}\right)\right]$ without evidence of the iminium salt. Finally, the chiral methylbenzylamine lead to the exclusive formation of compound $[\mathrm{Cp} * \operatorname{Ir}\{\mathrm{SC}(\mathrm{Me}) \mathrm{CHCH}(\mathrm{Me}) \mathrm{C}=\mathrm{N}(\mathrm{Me})(\mathrm{Bz})\}] \mathrm{BF}_{4}(\mathbf{1 7})$; however, several attempts to isolate it were unsuccessful and there was only ${ }^{1} \mathrm{H}$ and ${ }^{13} \mathrm{C}$ NMR spectroscopic evidence of its presence (Tables 1 and 2).

There was no reaction between compound $\mathbf{6}$ and diphenylamine, both of which were recovered, and there was no evidence of the iminium salt under the conditions described in the experimental section (vide infra) in the case of ephedrine, pyridine and aniline. Particularly, ephedrine was not a selective reaction. 
Table 1. ${ }^{1} \mathrm{H},{ }^{11} \mathrm{~B}$ and ${ }^{19} \mathrm{~F}$ NMR of compounds $\mathbf{1 2 - 1 7 ^ { \mathrm { a } }}$

\begin{tabular}{|c|c|c|c|c|c|c|c|c|}
\hline \multirow[t]{3}{*}{ Compound } & \multirow[t]{3}{*}{$\mathrm{S}-\mathrm{C}-\mathrm{Me}$} & \multirow[t]{3}{*}{$\mathrm{C} H_{\mathrm{A}}$} & \multirow[t]{3}{*}{$\mathrm{C} H_{\mathrm{B}}$} & \multirow[t]{3}{*}{$\mathrm{N}=\mathrm{CMe}$} & \multirow[t]{3}{*}{$\mathrm{N} R$} & \multirow[t]{3}{*}{$C p^{*}$} & \multirow{3}{*}{$\begin{array}{l}{ }^{11} \mathrm{~B} \&{ }^{19} \mathrm{~F} \\
\mathrm{NMR}\end{array}$} & \multirow{3}{*}{$\begin{array}{l}\text { Color } \\
\mathrm{Mp}\left({ }^{\circ} \mathrm{C}\right) \\
\text { Yield (\%) }\end{array}$} \\
\hline & & & & & & & & \\
\hline & & & & & & & & \\
\hline \multirow[t]{4}{*}{12} & $2.33(\mathrm{~s})$ & $6.11(\mathrm{~d}, 6.0)$ & $2.62(\mathrm{~d}, 6.0)$ & $2.29(\mathrm{~s})$ & $4.10(\mathrm{~m}, 2 \mathrm{H})$ & $1.95(\mathrm{~s})$ & $-1.68^{b}$ & Orange \\
\hline & & & & & $3.80(\mathrm{~m}, 4 \mathrm{H})$ & & $-153.1^{\mathrm{c}}$ & $178-180^{\mathrm{d}}$ \\
\hline & & & & & $3.45(\mathrm{~m}, 2 \mathrm{H})$ & & & 83.7 \\
\hline & & & & & $1.80(\mathrm{~m}, 2 \mathrm{H})$ & & & \\
\hline \multirow[t]{4}{*}{$13^{\mathrm{e}}$} & $2.33(\mathrm{~s})$ & $6.06(\mathrm{~d}, 6.2)$ & $2.47(\mathrm{~d}, 6.2)$ & $2.31(\mathrm{~s})$ & $3.80(\mathrm{t}, 2 \mathrm{H}, 6.5)$ & $1.92(\mathrm{~s})$ & $-1.79^{b}$ & Orange \\
\hline & & & & & $3.42(\mathrm{~s}, \mathrm{br}, 2 \mathrm{H})$ & & $-153.3^{\mathrm{c}}$ & $154-156$ \\
\hline & & & & & $3.20(\mathrm{~m}, 2 \mathrm{H})$ & & & 93.4 \\
\hline & & & & & $2.05(\mathrm{~m}, 2 \mathrm{H})$ & & & \\
\hline \multirow[t]{3}{*}{14} & $2.32(\mathrm{~s})$ & $6.20(\mathrm{~d}, 6.0)$ & $2.68(\mathrm{~d}, 6.0)$ & $2.31(\mathrm{~s})$ & $3.90(\mathrm{~m}, 4 \mathrm{H})$ & $1.97(\mathrm{~s})$ & $-1.67^{b}$ & Red \\
\hline & & & & & $3.70(\mathrm{~m}, 2 \mathrm{H})$ & & $-153.3^{\mathrm{c}}$ & 176-179 \\
\hline & & & & & $3.50(\mathrm{~m}, 2 \mathrm{H})$ & & & 86.7 \\
\hline \multirow[t]{3}{*}{15} & $2.31(\mathrm{~s})$ & $5.95(\mathrm{~d}, 6.2)$ & $2.56(\mathrm{~d}, 5.9)$ & $2.29(\mathrm{~s})$ & $3.94(\mathrm{~m}, 2 \mathrm{H})$ & $1.92(\mathrm{~s})$ & $-1.38^{\mathrm{b}}$ & Orange \\
\hline & & & & & $3.55(\mathrm{~m}, 2 \mathrm{H})$ & & $-151.8^{\mathrm{c}}$ & $140-143^{\mathrm{d}}$ \\
\hline & & & & & $3.06(\mathrm{~m}, 4 \mathrm{H})$ & & & 51.3 \\
\hline \multirow[t]{3}{*}{$15^{\mathrm{e}}$} & $2.33(\mathrm{~s})$ & $5.97(\mathrm{~d}, 6.2)$ & $2.64(\mathrm{~d}, 5.9)$ & $2.59(\mathrm{~s})$ & $3.87(\mathrm{~m}, 2 \mathrm{H})$ & $1.94(\mathrm{~s})$ & $-1.35^{\mathrm{b}}$ & \\
\hline & & & & & $3.60(\mathrm{~m}, 2 \mathrm{H})$ & & $-152.4^{\mathrm{c}}$ & \\
\hline & & & & & $3.05(\mathrm{~m}, 4 \mathrm{H})$ & & & \\
\hline \multirow[t]{3}{*}{16} & $2.33(\mathrm{~s})$ & $6.12(\mathrm{~d}, 6.1)$ & $2.62(\mathrm{~d}, 6.1)$ & $2.31(\mathrm{~s})$ & $3.80(\mathrm{~m}, 2 \mathrm{H})$ & $1.95(\mathrm{~s})$ & $-1.53^{b}$ & Orange \\
\hline & & & & & $3.55(\mathrm{~m}, 2 \mathrm{H})$ & & $-151.9^{c}$ & $178-179^{d}$ \\
\hline & & & & & $1.40(\mathrm{~m}, 6 \mathrm{H})$ & & & 47.8 \\
\hline \multirow[t]{4}{*}{$17^{\mathrm{f}}$} & $2.05(\mathrm{~s})$ & $6.20(\mathrm{~d}, 5.9)$ & $2.75(\mathrm{~d}, 6.4)$ & $2.24(\mathrm{~s})$ & $1.77(\mathrm{~d}, 6.9,3 \mathrm{H})$ & $1.60(\mathrm{~s})$ & $-1.94^{\mathrm{b}}$ & Not \\
\hline & & & & & $4.10(\mathrm{~m}, 1 \mathrm{H})$ & & $-146.9^{c}$ & isolated \\
\hline & & & & & $4.50(\mathrm{~m}, 1 \mathrm{H})$ & & & \\
\hline & & & & & $6.92-7.55(\mathrm{~m})$ & & & \\
\hline
\end{tabular}

(a) In ppm, J (Hz), $\mathrm{CD}_{3} \mathrm{NO}_{2}$; (b) ${ }^{11} \mathrm{~B}$; (c) ${ }^{19} \mathrm{~F}$; (d) Decompose; (e) In $\mathrm{CDCl}_{3}$; (f) In $\mathrm{C}_{6} \mathrm{D}_{6}$.

When a stoichiometric ratio of reactants was used, the reaction, monitored qualitatively by ${ }^{1} \mathrm{H}$ NMR, showed that no considerable conversion to products took place. Hence, it is clear that an excess of ligand is always necessary. The role of the basicity of the amine is not quite clear because piperidine and morpholine afford quite similar results. Apparently, steric effects may also prevent the nucleophilic addition reactions, as observed from the diphenylamine. 
Table 2. ${ }^{13} \mathrm{C}$ NMR, ${ }^{a}$ mass spectra and elemental analysis of compounds 12-17

\begin{tabular}{|c|c|c|c|c|c|c|c|c|c|c|}
\hline Compound & $\mathrm{S}-\mathrm{C}$ & $\mathrm{CH}_{\mathrm{A}}$ & $\mathrm{CH}_{\mathrm{B}}$ & $\mathrm{C}=\mathrm{N}$ & NR & $\begin{array}{l}\mathrm{Cp}^{*} \\
\mathrm{Cq}\end{array}$ & $\begin{array}{l}\mathrm{Cp}^{*} \\
\mathrm{Me}\end{array}$ & $\mathrm{S}-\mathrm{Me}$ & $\mathrm{C}-\mathrm{Me}$ & $\begin{array}{l}\mathrm{HRMS} \mathrm{m} / \mathrm{z} \quad\left[\mathrm{M}-\mathrm{BF}_{4}\right]^{+} \\
\text {or Elemental Analysis }\end{array}$ \\
\hline \multirow[t]{5}{*}{12} & 98.6 & 79.7 & 50.6 & 181.8 & 53.1 & 92.9 & 8.8 & 23.8 & 17.3 & $\mathrm{C}_{21} \mathrm{H}_{33} \mathrm{~S}_{1} \mathrm{Ir}_{1} \mathrm{~N}_{1}: 524.19629$ \\
\hline & & & & & 52.0 & & & & & Found: 524.19490 (error $=$ \\
\hline & & & & & 26.8 & & & & & $1.61 \mathrm{ppm})$ \\
\hline & & & & & 25.9 & & & & & \\
\hline & & & & & 23.2 & & & & & \\
\hline \multirow[t]{4}{*}{13} & 99.0 & 78.8 & 51.7 & 180.9 & 53.4 & 92.5 & 9.9 & 24.7 & 19.1 & $\mathrm{C}_{20} \mathrm{H}_{31} \mathrm{~S}_{1} \mathrm{Ir}_{1} \mathrm{~N}_{1}: 510.18064$ \\
\hline & & & & & 52.6 & & & & & Found: 510.18128 (error $=$ \\
\hline & & & & & 25.2 & & & & & $2.31 \mathrm{ppm})$ \\
\hline & & & & & 24.2 & & & & & \\
\hline \multirow[t]{4}{*}{14} & 99.6 & 79.8 & 51.1 & 183.4 & 66.4 & 93.0 & 8.8 & 23.8 & 17.4 & $\mathrm{C}_{20} \mathrm{H}_{31} \mathrm{~S}_{1} \mathrm{Ir}_{1} \mathrm{~N}_{1} \mathrm{O}_{1} \mathrm{BF}_{4}:$ \\
\hline & & & & & 65.8 & & & & & 612.54 theo/(found): \\
\hline & & & & & 51.6 & & & & & C: $39.22(39.20)$ \\
\hline & & & & & 50.4 & & & & & H: 5.10 (4.99) \\
\hline \multirow[t]{4}{*}{15} & 98.6 & 79.5 & 50.5 & 181.7 & 53.1 & 92.6 & 8.5 & 23.5 & 17.0 & $\mathrm{C}_{20} \mathrm{H}_{32} \mathrm{~S}_{1} \mathrm{Ir}_{1} \mathrm{~N}_{2}: 525.19154$ \\
\hline & & & & & 51.9 & & & & & Found: 525.19114 (error $=$ \\
\hline & & & & & 46.1 & & & & & $0.28 \mathrm{ppm})$ \\
\hline & & & & & 45.5 & & & & & \\
\hline \multirow[t]{4}{*}{$15^{\mathrm{b}}$} & 99.0 & 77.5 & 53.4 & 181.7 & 53.4 & 92.6 & 9.7 & 24.6 & 17.9 & \\
\hline & & & & & 51.7 & & & & & \\
\hline & & & & & 46.8 & & & & & \\
\hline & & & & & 46.0 & & & & & \\
\hline \multirow[t]{4}{*}{16} & 99.3 & 77.6 & 51.0 & 183.0 & 49.5 & 92.4 & 9.6 & 24.5 & 18.0 & $\mathrm{C}_{20} \mathrm{H}_{33} \mathrm{~S}_{1} \mathrm{Ir}_{1} \mathrm{~N}_{1} \mathrm{BF}_{4}: 598.56$ \\
\hline & & & & & 48.7 & & & & & theo/(found): \\
\hline & & & & & 13.2 & & & & & C: 40.13 (40.08) \\
\hline & & & & & 10.3 & & & & & H: 5.56 (5.79) \\
\hline \multirow[t]{6}{*}{$17^{\mathrm{c}}$} & 99.6 & 79.0 & 49.9 & 184.8 & 22.5 & 92.2 & 9.2 & 24.5 & 20.4 & Not isolated \\
\hline & & & & & 58.2 & & & & & \\
\hline & & & & & $140.7(\mathrm{i})$ & & & & & \\
\hline & & & & & $137.8(\mathrm{o})$ & & & & & \\
\hline & & & & & $129.6(p)$ & & & & & \\
\hline & & & & & $128.6(\mathrm{~m})$ & & & & & \\
\hline
\end{tabular}

a) In ppm, $\mathrm{CD}_{3} \mathrm{NO}_{2}$; b) In $\mathrm{CDCl}_{3}$; c) In $\mathrm{C}_{6} \mathrm{D}_{6}$.

The addition and C-S cleavage processes occur regiospecifically at the (Me)S-C linkage. The presence of only one isomer for derivatives 12-17 was established by ${ }^{1} \mathrm{H}$ and ${ }^{13} \mathrm{C}$ NMR which showed only one set of $\mathrm{Cp}^{*}$ signals. However, the reaction was monitored through ${ }^{1} \mathrm{H}$ NMR for 
compound 6 in presence of pyrrolidine, in a 1:2 ratio. This showed the formation of the kinetic product as the anti conformer $11{ }^{1} \mathrm{H} \mathrm{NMR}\left(\mathrm{CD}_{3} \mathrm{NO}_{2}\right) \delta: 5.62\left(\mathrm{~d}, 4.7 \mathrm{~Hz}, \mathrm{CH}_{\mathrm{A}}\right) ; 3.60\left(\mathrm{br}, \mathrm{CH}_{\mathrm{B}}\right)$; $\left.2.34(\mathrm{~s}, \mathrm{Me}) 2.00\left(\mathrm{~s}, \mathrm{Cp}^{*}\right)\right]$ which easily went to the corresponding syn thermodynamic one $\mathbf{1 3}$

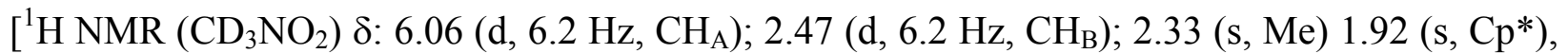
Table 1] (Scheme 2). Additional to the ${ }^{1} \mathrm{H}$ and ${ }^{13} \mathrm{C}$ NMR spectra of 12-17, HETCOR and APT experiments were performed to support the corresponding assignment. The ${ }^{11} \mathrm{~B}$ and ${ }^{19} \mathrm{~F}$ NMR confirmed the presence of the $\mathrm{BF}_{4}$ counterion in all cases.

The iminum carbon is demonstrably electrophilic, because it is susceptible to base hydrolysis attack, as observed for ruthenium analogue species. ${ }^{1}$ Partial recyclization of the thiophene ligand proceeds in the thermodynamic compound $\mathbf{1 2}$ in presence of triflic acid. It is known that the kinetic isomers of the ruthenium analogues are rapidly transformed after treatment with protic sources. ${ }^{1}$ The iridium compound $\mathbf{1 3}$ showed faster interconversion between kinetic and thermodynamic products, which did not allow to compare the reactivity of the kinetic isomer with the ruthenium analogue.

Attempts to remove the $\mathrm{Cp} * \mathrm{Ir}$ fragment from compound $\mathbf{1 3}$ were carried out in presence of $\left(\mathrm{n}-\mathrm{Bu}_{4}\right) \mathrm{NCl}$ or MeOTf unsuccessfully, which suggest the high stability of the $\eta^{3}$-allyl-Ir bond. Addition of $\mathrm{D}_{2} \mathrm{O}$ to compound $\mathbf{1 2}$ did not show any change even after a week; meanwhile an equimolar water solution of compound 13 and $\mathrm{H}_{2} \mathrm{O} / \mathrm{KOH}(0.03 \mathrm{M})$ afforded quantitatively the acylthiolate 7. Compounds 12-14 melted, 13 and 14 without decomposition, and crystallized from nitromethane/diethyl ether as single crystals, except $\mathbf{1 3}$ which crystallized from $\mathrm{CHCl}_{3}$ at room temperature. The crystallographic studies were carried out at room temperature and the assignment of these species as an iminium derivative is supported by the bond distances and angles at $\mathrm{C} 4$. The $\mathrm{C}=\mathrm{N}$ distances of 12, 13 and $\mathbf{1 4}$ are 1.313(6), 1.306(12) and 1.308(10) $\AA$, respectively, which is appropiate for a double bond, whereas bonds between nitrogen and the carbon atoms of the corresponding cyclic amines are longer N-C7 1.491(6), N-C11 1.494(7) 12; N-C7 1.464(12), N-C10 1.441(16) 13; and N-C7 1.479(11), N-C10 1.477(10) $14 \AA$. The molecular structures are presented in Figures 1-3, respectively. Table 3 includes the corresponding crystal data and Tables 4 and 5 selected bond lengths and bond angles, respectively.

The structure of these iminium salts in the solid state revealed a sandwich structure wherein the thiophene ligand is cleaved. These iminium centers are conjugated via a $\pi$-bonding network with the rest of the allylthiolate, where the highest delocalization was observed for compound $\mathbf{1 2}$, and similar behavior has been reported for the aminolysis of dicationic ruthenium thiophene compounds. ${ }^{1}$

The distances of $\operatorname{Ir}(1)-\mathrm{S}(1)$ and $\mathrm{S}(1)-\mathrm{C}(1)$ are decreasing in the following order: $\mathbf{1 2}>\mathbf{1 4}>\mathbf{1 3}$ and $14>12>13$, respectively. The piperidine and morpholine fragments showed a chair conformation and the pyrrolidine, an envelope one. 
Table 3. X-ray data collection parameters for compounds 12-14

\begin{tabular}{|c|c|c|c|}
\hline Compound & 12 & 13 & 14 \\
\hline formula & $\mathrm{C}_{21} \mathrm{H}_{33} \mathrm{BF}_{4} \operatorname{IrNS}$ & $\mathrm{C}_{21} \mathrm{H}_{32} \mathrm{BCl}_{3} \mathrm{~F}_{4} \mathrm{IrNS}$ & $\mathrm{C}_{20} \mathrm{H}_{31} \mathrm{BF}_{4} \mathrm{IrNOS}$ \\
\hline $\mathrm{fw}$ & 610.55 & 715.90 & 612.53 \\
\hline Cryst syst & Monoclinic & Triclinic & Triclinic \\
\hline Space Group & $\mathrm{P} 2 / \mathrm{c}$ & $\mathrm{P}-1$ & $\mathrm{P}-1$ \\
\hline $\mathrm{a}(\AA)$ & $13.450(3)$ & $9.6363(10)$ & $8.3432(2)$ \\
\hline $\mathrm{b}(\AA)$ & $7.877(3)$ & $12.0380(10)$ & $11.4873(3)$ \\
\hline c $(\AA)$ & $21.840(3)$ & $12.6614(10)$ & $12.5263(4)$ \\
\hline$\alpha\left({ }^{\circ}\right)$ & 90 & $67.321(10)$ & $95.8270(10)$ \\
\hline$\beta\left({ }^{\circ}\right)$ & $91.34(2)$ & $74.241(10)$ & $108.9380(1)$ \\
\hline$\gamma\left({ }^{\circ}\right)$ & 90 & $85.072(10)$ & $97.3640(10)$ \\
\hline $\mathrm{V}\left(\AA^{3}\right)$ & $2313.2(11)$ & $1304.0(2)$ & $1113.00(5)$ \\
\hline Z & 4 & 2 & 2 \\
\hline $\mathrm{D}_{\text {calc }}\left(\mathrm{g} \mathrm{cm}^{-3}\right)$ & 1.753 & 1.823 & 1.828 \\
\hline Temp (K) & $270(2)$ & $293(2)$ & $298(2)$ \\
\hline Diffractometer & $\begin{array}{l}\text { Enraf Nonius } \\
\text { CAD4 }\end{array}$ & $\begin{array}{l}\text { Enraf-Nonius } \\
\text { CAD4 }\end{array}$ & Kappa CCD \\
\hline $2 \theta$ scan range $\left({ }^{\circ}\right)$ & 5.18 to $51.92^{\circ}$ & 5.32 to 52.48 & 5.84 to 55.08 \\
\hline \multirow[t]{3}{*}{ Index ranges } & $-16 \leq h \leq 16$ & $-11 \leq \mathrm{h} \leq 11$ & $-10 \leq h \leq 10$ \\
\hline & $0 \leq k \leq 9$ & $-13 \leq \mathrm{k} \leq 14$ & $-12 \leq k \leq 14$ \\
\hline & $0 \leq l \leq 26$ & $0 \leq 1 \leq 15$ & $-16 \leq l \leq 14$ \\
\hline no. of reflns collcd & 4527 & 2932 & 10670 \\
\hline no. of indpt reflns & 4527 & 2932 & 5058 \\
\hline no. of indpt obsd & $3635(\mathrm{~F}>4 \sigma(\mathrm{F}))$ & $2932(\mathrm{~F}>4 \sigma(\mathrm{F}))$ & $4204(\mathrm{~F}>4 \sigma(\mathrm{F}))$ \\
\hline Final $R_{1}$ & 0.0282 & 0.0391 & 0.0560 \\
\hline Final $w_{2}$ & 0.0801 & 0.1037 & 0.1253 \\
\hline GOOF & 1.081 & 1.056 & 1.070 \\
\hline
\end{tabular}


Table 4. Selected bond lengths ( $\AA$ ) for compounds 12-14

\begin{tabular}{|c|c|c|c|c|}
\hline Compound & 12 & & 13 & 14 \\
\hline $\operatorname{Ir}(1)-S(1)$ & $2.4066(15)$ & & $2.372(3)$ & $2.393(2)$ \\
\hline $\operatorname{Ir}(1)-C(1)$ & $2.173(5)$ & & $2.146(7)$ & $2.158(8)$ \\
\hline $\operatorname{Ir}(1)-C(2)$ & $2.137(4)$ & & $2.138(7)$ & $2.145(7)$ \\
\hline $\operatorname{Ir}(1)-C(3)$ & $2.182(5)$ & & $2.178(9)$ & $2.191(8)$ \\
\hline$S(1)-C(1)$ & $1.750(5)$ & & $1.734(11)$ & $1.755(9)$ \\
\hline$C(1)-C(2)$ & $1.417(7)$ & & $1.422(14)$ & $1.404(11)$ \\
\hline$C(2)-C(3)$ & $1.423(7)$ & & $1.465(13)$ & $1.457(11)$ \\
\hline$C(3)-C(4)$ & $1.438(7)$ & & $1.426(14)$ & $1.436(11)$ \\
\hline $\mathrm{C}(4)-\mathrm{N}(1)$ & $1.313(6)$ & & $1.306(12)$ & $1.308(10)$ \\
\hline$C(4)-C(5)$ & $1.488(7)$ & & $1.474(17)$ & $1.502(12)$ \\
\hline$C(1)-C(6)$ & $1.500(7)$ & & $1.480(14)$ & $1.505(11)$ \\
\hline $\mathrm{N}(1)-\mathrm{C}(7)$ & $1.491(6)$ & & $1.464(12)$ & $1.479(11)$ \\
\hline$C(7)-C(8)$ & $1.537(8)$ & & $1.51(2)$ & $1.507(14)$ \\
\hline$C(9)-C(10)$ & $1.493(11)$ & & $1.525(15)$ & $1.517(13)$ \\
\hline$C(8)-C(9)$ & $1.514(9)$ & & $1.496(18)$ & \\
\hline$C(10)-C(11)$ & $1.501(9)$ & & & \\
\hline $\mathrm{N}(1)-\mathrm{C}(11)$ & $1.494(7)$ & $\mathrm{N}(1)-\mathrm{C}(10)$ & $1.441(16)$ & $1.477(10)$ \\
\hline $\operatorname{Ir}(1)-C(12)$ & $2.179(5)$ & $\operatorname{Ir}(1)-C(11)$ & $2.169(9)$ & $2.158(8)$ \\
\hline $\mathrm{C}(8)-\mathrm{O}(1)$ & & & & $1.423(11)$ \\
\hline $\mathrm{C}(9)-\mathrm{O}(1)$ & & & & $1.415(11)$ \\
\hline
\end{tabular}

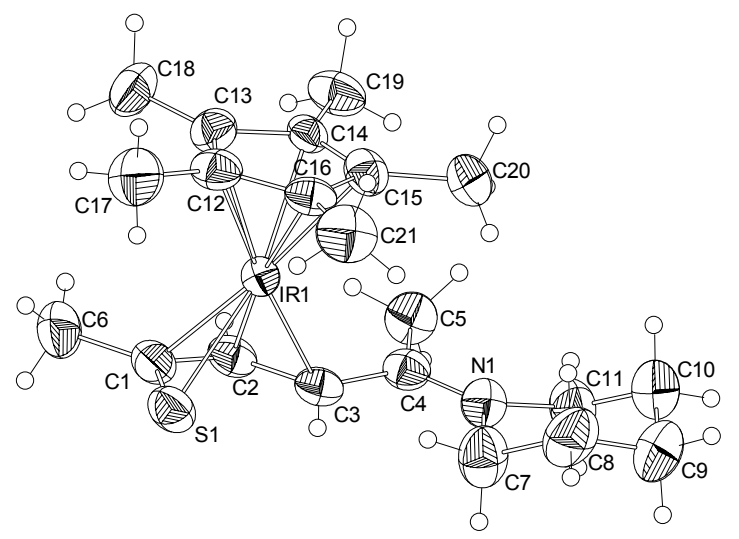

Figure 1. ORTEP drawing of the cationic portion of compound 12. 
Table 5. Selected bond angles (deg) for compounds 12-14

\begin{tabular}{llll}
\hline Compound & $\mathbf{2}$ & $\mathbf{1 3}$ & $\mathbf{1 4}$ \\
\hline $\mathrm{C}(1)-\operatorname{Ir}(1)-\mathrm{S}(1)$ & $44.57(13)$ & $44.8(3)$ & $45.0(2)$ \\
$\mathrm{C}(2)-\operatorname{Ir}(1)-\mathrm{C}(1)$ & $38.39(19)$ & $38.8(4)$ & $38.1(3)$ \\
$\mathrm{C}(2)-\operatorname{Ir}(1)-\mathrm{S}(1)$ & $72.69(14)$ & $73.1(3)$ & $73.2(2)$ \\
$\mathrm{C}(3)-\operatorname{Ir}(1)-\mathrm{S}(1)$ & $77.59(15)$ & $77.2(3))$ & $77.4(2)$ \\
$\mathrm{Ir}(1)-\mathrm{S}(1)-\mathrm{C}(1)$ & $60.63(16)$ & $60.7(3)$ & $60.4(3)$ \\
$\mathrm{C}(1)-\mathrm{C}(2)-\mathrm{C}(3)$ & $117.7(4)$ & $115.6(10)$ & $116.0(7)$ \\
$\mathrm{C}(2)-\mathrm{C}(3)-\mathrm{C}(4)$ & $124.0(5)$ & $121.2(12)$ & $123.2(7)$ \\
$\mathrm{C}(3)-\mathrm{C}(4)-\mathrm{C}(5)$ & $120.3(5)$ & $124.2(9)$ & $119.5(7)$ \\
$\mathrm{C}(4)-\mathrm{N}(1)-\mathrm{C}(7)$ & $121.0(4)$ & $124.4(10)$ & $123.8(7)$ \\
$\mathrm{N}(1)-\mathrm{C}(4)-\mathrm{C}(3)$ & $120.8(4)$ & $116.1(10)$ & $121.3(7)$ \\
$\mathrm{C}(2)-\mathrm{C}(1)-\mathrm{S}(1)$ & $116.7(4)$ & $116.7(7)$ & $117.8(6)$ \\
$\mathrm{C}(2)-\mathrm{C}(1)-\operatorname{Ir}(1)$ & $69.4(3)$ & $70.3(4)$ & $70.4(4)$ \\
$\mathrm{S}(1)-\mathrm{C}(1)-\operatorname{Ir}(1)$ & $74.80(18)$ & $74.5(3)$ & $74.6(3)$ \\
& & & \\
$\mathrm{C}(1)-\mathrm{C}(2)-\operatorname{Ir}(1)$ & $72.2(3)$ & $70.9(4)$ & $71.5(5)$ \\
$\mathrm{C}(4)-\mathrm{N}(1)-\mathrm{C}(11)$ & $122.8(5)$ & & \\
$\mathrm{C}(11)-\mathrm{N}(1)-\mathrm{C}(7)$ & $115.2(5)$ & & \\
\hline
\end{tabular}

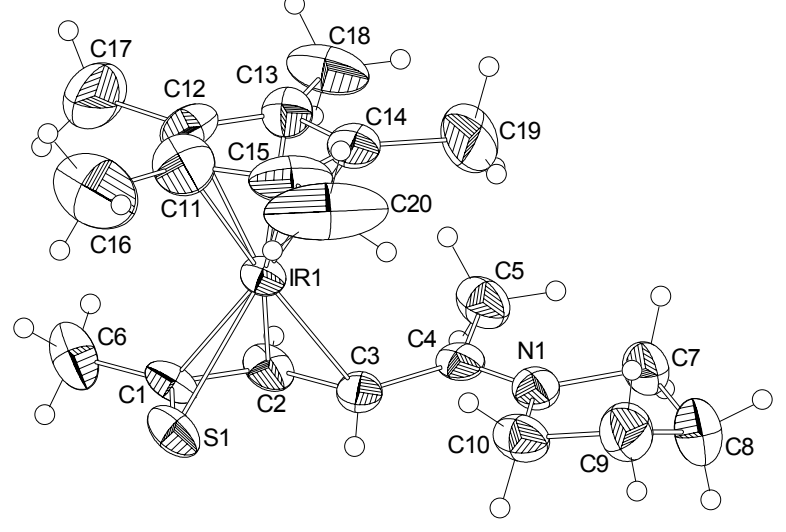

Figure 2. ORTEP drawing of the cationic portion of compound 13. 


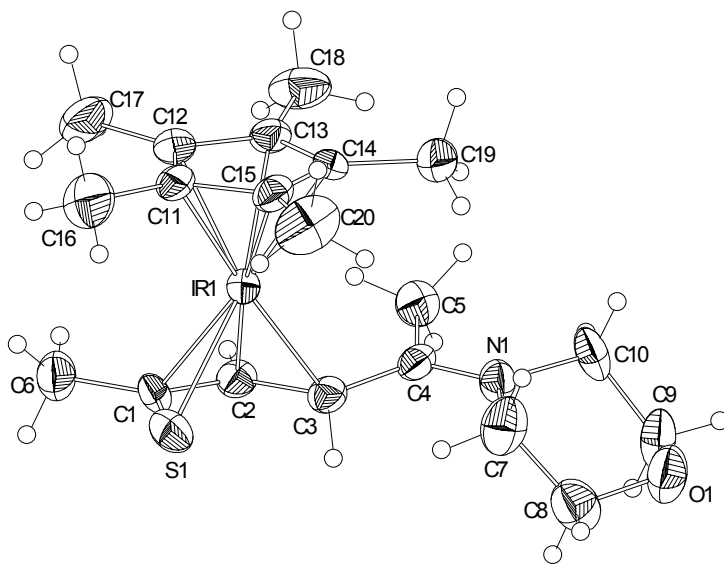

Figure 3. ORTEP drawing of the cationic portion of compound $\mathbf{1 4}$

The IR spectra of 12-16 shows a strong band, assigned to the $v_{\mathrm{C}=\mathrm{N}}$, at around $\left(\mathrm{cm}^{-1}\right): 1578, \mathbf{1 2}$; 1594, 13; 1571, 14; 1574, 15 and 1590, 16.

\section{Experimental Section}

General Procedures. All reactions were carried out with exclusion of air using Schlenk-tube techniques. Solvents were dried by the usual procedures and distilled under nitrogen prior to use. The starting material $\mathrm{Cp} * \operatorname{Ir}\left(2,5-\right.$ dimethyl- $\eta^{5}$-thiophene $\left.)\right]\left[\mathrm{BF}_{4}\right]_{2}(\mathbf{6})$ was prepared by the published method. ${ }^{5}$

In the ${ }^{1} \mathrm{H}$ and ${ }^{13} \mathrm{C},{ }^{11} \mathrm{~B}$ and ${ }^{19} \mathrm{~F}$ NMR spectra, chemical shifts are expressed in ppm downfield from $\mathrm{Me}_{4} \mathrm{Si}, \mathrm{BF}_{3} \cdot \mathrm{OEt}_{2}$ and $\mathrm{CF}_{3} \mathrm{COOH}$, respectively. Coupling constants, J, are given in Hertz.

Preparation of Compounds $\left[\mathrm{Cp} * \operatorname{Ir}\left\{\mathrm{SC}(\mathrm{Me}) \mathrm{CHCH}(\mathrm{Me}) \mathrm{C}=\mathrm{NR}_{2}\right\}\right] \mathrm{BF}_{4}\left[\mathrm{R}_{2}=\right.$ piperidyl (12); $\mathbf{R}_{2}=$ pyrrolidyl (13); $\mathbf{R}_{2}=$ morpholyl (14); $R_{2}=$ piperazyl (15); $R=$ diethyl (16); $R=$ methyl, benzyl (17). General procedure

The addition of an excess of the amine (2 equiv) in $\sim 1-2 \mathrm{~mL}$ of $\mathrm{CH}_{3} \mathrm{NO}_{2}$ to a solution of compound 6 (150 mg, $0.245 \mathrm{mmol})$ in $\mathrm{CH}_{3} \mathrm{NO}_{2}(30 \mathrm{~mL})$ was stirred at room temperature for 18 $\mathrm{h}$. Immediately after the addition of the amine, a strong change in color was observed (vide infra). The solvent was evaporated and the product was extracted with $\sim 30 \mathrm{~mL}$ of $\mathrm{CH}_{2} \mathrm{Cl}_{2}$, the volume was reduced to $\sim 10 \mathrm{~mL}$ and precipitated with $\mathrm{Et}_{2} \mathrm{O}(\sim 60 \mathrm{~mL})$. The corresponding solids of 12 and 14 were recrystallized from $\mathrm{CH}_{3} \mathrm{NO}_{2} / \mathrm{Et}_{2} \mathrm{O}$, at $-10{ }^{\circ} \mathrm{C}$, to afford powders or single crystals. Compound 13 was recrystallized from $\mathrm{CHCl}_{3}$ at room temperature. See Table 1 and 2 for data related to melting point, elemental analyses, yield, mass spectra and NMR spectroscopy. The different compounds were:

$\mathbf{R}_{\mathbf{2}}=$ piperidyl (12). The orange solution afforded orange crystals. 
$\mathbf{R}_{\mathbf{2}}=$ pyrrolidyl(13). The orange solution afforded red crystals.

$\mathbf{R}_{\mathbf{2}}=$ morpholyl (14). The orange-dark solution afforded cherry-red crystals.

$\mathbf{R}_{\mathbf{2}}=$ piperazyl (15). The orange-red solution afforded an orange-red solid of $\mathbf{1 5}$ along with an unknown orange compound in a 6:4 ratio, respectively.

$\mathbf{R}=$ diethyl (16). The yellow-brown solution afforded a brown-orange solid, which after recrystallization with $\mathrm{Et}_{2} \mathrm{O}$ gave an insoluble white powder corresponding to the quaternary salt $\left[\mathrm{NH}_{2}(\mathrm{Et})_{2}\right] \mathrm{BF}_{4}$ in $47.8 \%$ yield (related to the amine), ${ }^{6}\left[{ }^{1} \mathrm{H} \mathrm{NMR}\left(\mathrm{CDCl}_{3}\right) \delta: 6.8(\mathrm{~s}, \mathrm{br}) ; 3.14(\mathrm{~m})\right.$; 1.35(m). ${ }^{13} \mathrm{C} \mathrm{NMR}\left(\mathrm{CDCl}_{3}\right) \delta: 44.0\left(\mathrm{CH}_{2}\right)$ and $\left.11.2(\mathrm{Me})\right]$; meanwhile in the soluble fraction of the remaining solid, the acylthiolate iridium compound 7 was isolated in $44.9 \%$ yield (related to compound 6). ${ }^{3,4}$ A chromatographic column of alumina was carried out for separation of both cationic compounds in $\mathrm{CH}_{2} \mathrm{Cl}_{2}$; the quaternary salt stayed at the top of the column and compound 6 was eluted with $\mathrm{CH}_{2} \mathrm{Cl}_{2}$ /acetone to afford an orange oil which after recrystallization in $\mathrm{CHCl}_{3} / \mathrm{Et}_{2} \mathrm{O}$ gave an orange solid.

$\mathbf{R}=$ methyl, benzyl (17). In our hands, it was not possible to isolate this product. However, according with ${ }^{1} \mathrm{H}$ and ${ }^{13} \mathrm{C}$ NMR spectroscopy this is the only product formed during the reaction. Attempts to isolate $\mathbf{1 7}$ led only to unidentified decomposition products.

$\mathrm{R}=$ diisopropyl The yellow-green solution afforded a yellow solid which after purification afforded the quaternary salt of $\left[\mathrm{NH}_{2}(\mathrm{CHMe})_{2}\right] \mathrm{BF}_{4}$ in $52.3 \%$ yield (related to the amine) $\left[{ }^{1} \mathrm{H}\right.$ NMR (acetone- $\mathrm{d}_{6}$ ) $\delta: 7.1$ (s, br); 3.71 (septuplete); 1.40(d, $\left.6.7 \mathrm{~Hz}\right) .{ }^{13} \mathrm{C}$ NMR $\delta: 48.4(\mathrm{CH}) ; 18.4$ (Me). ${ }^{11} \mathrm{~B}$ NMR $\delta:-1.62 .{ }^{19} \mathrm{~F}$ NMR $\delta$ : - 146.8] and compound 7, (59\%) (related to compound 6). Both products were isolated from several recystallizations of $\mathrm{CH}_{2} \mathrm{Cl}_{2} / \mathrm{Et}_{2} \mathrm{O}$ and $\mathrm{Et}_{2} \mathrm{O}$, respectively.

$\mathrm{R}=$ dicyclohexyl The amber solution afforded a yellow-oily solid which after purification afforded the quaternary salt of $\left[\mathrm{NH}_{2}\left(\mathrm{C}_{6} \mathrm{H}_{11}\right)_{2}\right] \mathrm{BF}_{4}(54 \%)$ (related to the amine). [ ${ }^{1} \mathrm{H} \mathrm{NMR}$ $\left(\mathrm{CDCl}_{3}\right)$ \&: 6.4 (s, br); $3.11(\mathrm{~m}) ; 1.21-2.07(\mathrm{~m}) .{ }^{13} \mathrm{C} \mathrm{NMR} \delta: 54.8(\mathrm{CH}) ; 29.15,24.59\left(\mathrm{CH}_{2}\right) ;{ }^{11} \mathrm{~B}$ NMR $\delta$ : $-1.67 ;{ }^{19}$ F NMR $\delta$ : -146.7 ] and compound 7 (56\%) (related to compound 6).

\section{$\underline{\text { Supplementary Information Available }}$}

Crystal data, tables of atom coordinates and tempertaure factors, bond lengths, bond angles, anisotropic temperature factors, torsion angles and labeled ORTEP diagrams (43 pages). CCDC 659916, 659915 and 659914 contain the supplementary crystallographic data for 12, 13 and 14.

\section{Acknowledgements}

This research was supported by Conacyt through project No. 38507 E and 46556 Q and B. P. M. thanks Conacyt for a research studentship. 


\section{References}

1. Feng, Q.; Rauchfuss, B.; Wilson, S. R., Organometallics 1995, 14, 2923.

2. Krautscheid, H.; Feng, Q.; Rauchfuss, T. B. Organometallics 1993, 12, 3273.

3. Paz-Sandoval, M. A.; Cervantes-Vasquez, M.; Young, V. G. Jr.; Guzei, I. A.; Angelici, R. J. Organometallics 2004, 23, 1274.

4. Cheng, J.; Su, Y.; Jacobson, R. A.; Angelici, R. J. J. Organometal. Chem. 1996, 512, 149.

5. Cheng, J.; Daniels, L. M.; Angelici R.J. J. Am. Chem. Soc. 1990, 112, 199.

6. Zabinska, G.; Ferloni, P.; Sanesi, M. Thermochimica Acta 1988, 137, 39. 